\title{
Hypersonic Separated Flows about “Tick” Configurations with Sensitivity to Model Design
}

\author{
J. N. Moss' ${ }^{\mathrm{a}}$ S. O’Byrne ${ }^{\mathrm{b}}$, and S. L. Gai ${ }^{\mathrm{b}}$ \\ ${ }^{a}$ Mail Stop 408A, NASA Langley Research Center, Hampton, VA 23681-0001, USA \\ ${ }^{b}$ School of Engineering \& IT, University of NSW, Canberra, ACT, 2602, Australia
}

\begin{abstract}
This paper presents computational results obtained by applying the direct simulation Monte Carlo (DSMC) method for hypersonic nonequilibrium flow about "tick-shaped" model configurations. These test models produces a complex flow where the nonequilibrium and rarefied aspects of the flow are initially enhanced as the flow passes over an expansion surface, and then the flow encounters a compression surface that can induce flow separation. The resulting flow is such that meaningful numerical simulations must have the capability to account for a significant range of rarefaction effects; hence the application of the DSMC method in the current study as the flow spans several flow regimes, including transitional, slip, and continuum. The current focus is to examine the sensitivity of both the model surface response (heating, friction and pressure) and flowfield structure to assumptions regarding surface boundary conditions and more extensively the impact of model design as influenced by leading edge configuration as well as the geometrical features of the expansion and compression surfaces. Numerical results indicate a strong sensitivity to both the extent of the leading edge sharpness and the magnitude of the leading edge bevel angle. Also, the length of the expansion surface for a fixed compression surface has a significant impact on the extent of separated flow.
\end{abstract}

Keywords: DSMC, Leading Edge Effects, Hypersonic Flow, Separated Flow, Surface Effects, Rarefaction Effects PACS: $51.20 .+\mathrm{d}, 51.10 .+\mathrm{y}$

\section{INTRODUCTION}

Motivation for the current pretest DSMC simulations is to provide insight as to the experimental environment enveloping models when tested in the T-ADFA free-piston shock tunnel located at The University of NSW, Canberra, Australia, and for enhancing the experimental design to provide high-quality, benchmark data of hypersonic separated flows. Because of the complex nature of hypersonic separated flows and their attendant shockshock interactions, they continue to receive considerable attention because of their critical impact on the performance and design of hypersonic vehicles. Consequently, there is a critical need for benchmark experiments to validate computational models and tools to be used for specific hypersonic conditions encountered in various applications, particularly entry and re-entry applications. By means of an integrated application of theoretical, experimental, and computational assets, advancements in our ability to predict the occurrence and impact of separated flows should occur.

In addition to the normal surface measurements for pressure and heating rate, planar laser-induced fluorescence measurements of velocity and temperature will be a major focus of the experimental campaign being organized at The University of NSW, Canberra, to provide a much needed basis for relating the thermochemical state of the flow with the test model surface response. Such detailed data has been elusive as evident by the absence of both surface flux measurements and the attendant details concerning the thermochemical state of the flow.

The present paper expands on the DSMC results previously presented and compared with Navier-Stokes computations [1, 2], where two nominal T-ADFA flow conditions were investigated: (1) Condition A with significant rarefaction effects and where substantial differences [1,2] were observed between the DSMC and Navier- Stokes (without slip effects) simulations and (2) Condition E [2] at a higher density flow (approximately three times higher). Understandably, the agreement [2] was much improved between the two computational approaches relative to that for Condition A, the more rarefied case. Hence, the current paper focuses exclusively on Condition A, and it highlights the sensitivity of the current DSMC results to wall temperature, surface accommodation of vibrational energy, leading edge configuration (sharp versus slightly rounded), and leading edge bevel angle (attached versus detached flow) on flowfield structure and surface flux. Recently, an experimental test model has been fabricated where the geometrical features of the test model are somewhat different from that used in the preliminary studies and where the length of the expansion surface can be varied for a fixed compression surface. Results are presented that demonstrate the impact of such model changes on flow field structure and surface flux. 


\section{MODEL AND FLOW CONDITIONS}

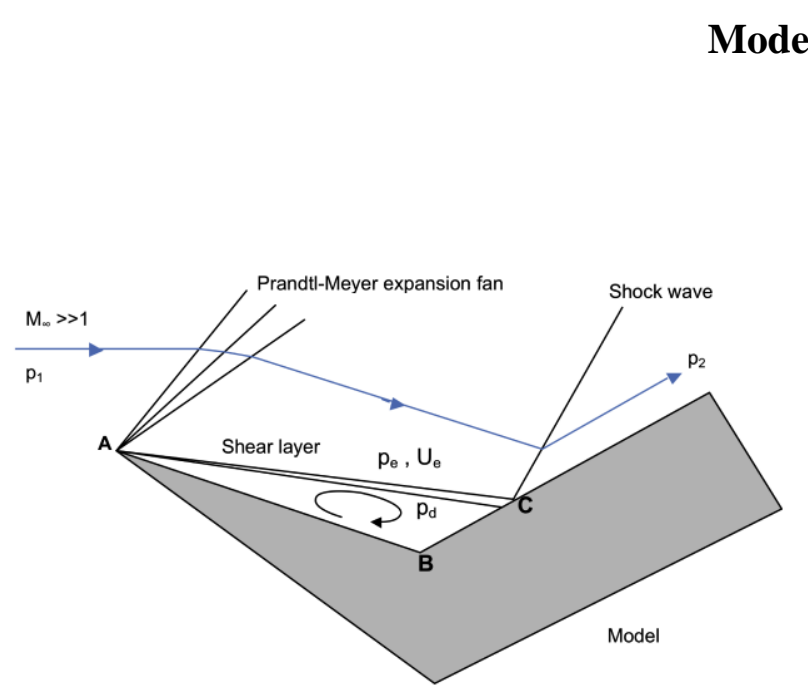

(a)

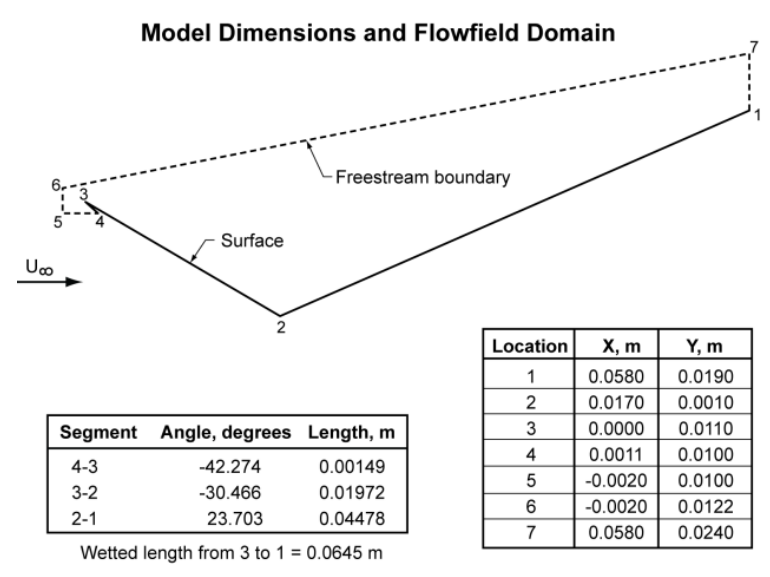

(b)

FIGURE 1. (a) Geometry of the 'tick’ configuration, (b) Example of computational domain geometry

Figure 1(a) shows the 'tick' configuration considered in this paper, where the model is two dimensional. This model geometry is equivalent to the 'leading-edge separation' model discussed in Chapman et al. [3], and consists of a sharp leading edge $\mathrm{A}$, with a downward sloping surface, terminating in a corner at B. The flow separates between $\mathrm{A}$ and $\mathrm{B}$, and re-attaches to the model surface at the point $\mathrm{C}$. As previously discussed $[1,2]$, the location of separation is strongly dependent on the flow rarefaction and leading edge sharpness. Figure 1 (b) shows the geometry for one of the sharp leading edge models used in the DSMC simulations. The region below the leading edge was truncated, as there is no interest in that region of the flow, except insofar as it influences the flow around corner A. Note that different lengths of truncation were explored for this configuration, and that shown in Fig. 1(b) is believed to be adequate to capture forebody disturbances. However, if the inflow boundary is placed at the tip of the sharp leading edge, it has a profound effect on the overall flow field, particularly along the expansion surface as will be demonstrated later.

\section{Flow Conditions}

The freestream conditions used for this study were taken from previous investigations in the T-ADFA free-piston shock tunnel at the University of NSW, Canberra $[4,5]$ for the flow around a blunt entry vehicle configuration. This facility uses piston compression to generate a high-speed shock that reflects at the entrance to a convergingdiverging nozzle. The flow conditions at the nozzle exit were calculated based upon measured pressure in the nozzle plenum and the shock speed in the shock tube. The measured initial shock speed and nozzle reservoir pressure were used to predict the conditions after the shock reflection at the end of the shock tube, using the chemical equilibrium shock tube code ESTC [6]. The expansion of the flow in the nozzle is computed using the one-dimensional inviscid chemical nonequilibrium code STUBE [7], modified to account for vibrational nonequilibrium using the sudden freezing model of Bray [8] for the molecular species $\mathrm{N}_{2}, \mathrm{O}_{2}$, and NO. In experiments, the NO produced in the shock reflection process is used for planar laser-induced fluorescence measurements of velocity and temperature [4, 5].

The freestream flow conditions used in the simulations for Condition A were: velocity $=3,730 \mathrm{~m} / \mathrm{s}$, number density $=4.61 \times 10^{22} \mathrm{~m}^{-3}\left(\right.$ density $\left.=0.0020 \mathrm{~kg} / \mathrm{m}^{3}\right)$, translational and rotational temperature $=593 \mathrm{~K}$ and vibrational temperatures for $\mathrm{N}_{2}=3,400 \mathrm{~K}, \mathrm{O}_{2}=2,070 \mathrm{~K}$ and $\mathrm{NO}=760 \mathrm{~K}$. The nozzle exit mole fractions were: $\mathrm{N}_{2}=0.690$, $\mathrm{O}_{2}=0.075$, $\mathrm{NO}=0.045$ and $\mathrm{O}=0.19$. As evident, the flow is in vibrational nonequilibrium for all molecular species, with vibrational temperatures elevated above the translational/rotational temperature at the nozzle exit. 


\section{COMPUTATIONAL MODEL}

The DSMC calculations were performed using the DS2V code of Bird, a general-purpose 2D/axisymmetric code that provides both time-accurate unsteady flow and time-averaged steady flow simulations. Details concerning physical aspects of the method are discussed in Ref. [9] while specific features of the code are described in Ref. [10]. For this study, the simulations are performed with chemically nonreacting and reacting gas models, while considering energy exchange between translation, rotational, and vibrational internal energy modes. The surface is assumed to be non-catalytic and at a specified wall temperature of either $300 \mathrm{~K}$ (a baseline condition that is likely to be encountered in a pulsed facility such as T-ADFA) or 1,000 $\mathrm{K}$ to assess the importance of a more realistic hypersonic hot wall. As for the gas-surface interactions, two models are assumed: one in which all energy modes are fully accommodated (baseline condition) and the other in which all energy modes except for vibration are fully accommodated.

An indication of the resolution achieved in a DS2V simulation is provided by the local ratio of the mean collision separation between collision partners to the local mean free path (mcs/mfp). As demonstrated in Ref. [11] for shock interaction problems with separation, it is desirable that $\mathrm{mcs} / \mathrm{mfp}$ be less than unity throughout the computational domain. In the current investigation, grid sensitivity assessments are made that identify specific requirements in terms of simulated molecules and collision cells that provide grid-independent results. For Condition A, the total number of molecules modeled was approximately 9.2 million, and approximately 1 million collision cells were used. At these conditions, the maximum value of the mcs/mfp parameter was 0.70 with an average value of 0.13 , which satisfies the requirement mentioned above.

\section{RESULTS AND DISCUSSION}

The initial discussion expands on the results presented in Refs. [1, 2] by briefly summarizing those results and presenting additional information as to the effect of altering the surface temperature and surface accommodation for the vibrational energy using the identical 'tick' configuration shown in Fig. 1. These results will then be followed with new data obtained for 'tick' configurations for which test models have been fabricated for future experiments..

\section{Effect of Surface Temperature and Surface Vibrational Accommodation}

As discussed in Refs. [1, 2], the DSMC simulations for Condition A and for the initial sharp leading edge 'tick' configuration produced a flow with essentially no separation while the perfect gas Navier-Stokes simulation, without including the effects of slip, produced a sizable separated region. Examination of the local Knudsen numbers (using local values for density, density gradients, and mean free path) throughout the computational domain indicated that the values ranged from about 0.1 to 10 ; that is, flow for which significant rarefaction is present for much of the flow - very much evident over the expanding surface. Evidence of the impact of rarefaction along the initial expansion surface is found in the large values for slip resulting from the DSMC simulations: maximum values for velocity slip of about $950 \mathrm{~m} / \mathrm{s}$ and temperature of about 1,900 K. Also, there was clear evidence of species separation over the expansion surface. For example, there was approximately a factor of two increase in the mole fraction of atomic oxygen with respect to its freestream value over and near the expansion surface, while there was a decrease in the concentration of the heavier species. Consequently, the substantial differences observed between the two simulation methods for both the surface flux and flow structure is not unexpected for Condition A.

To assess the importance of a hot wall boundary condition, which is more representative of flight conditions than a room-temperature wall boundary condition, simulations were made for a constant wall temperature of 1,000 K. The sharp leading edge results for this simulation are presented in Fig. 2, where the surface heating rate and skin friction results are compared with the equivalent values for a wall temperature of $300 \mathrm{~K}$. These simulations were made with a five species reacting air gas model, even though selective testing indicated that the results were essentially the same when one used a nonreacting four species gas model (deleting atomic nitrogen).

Normally an increase in wall temperature results in a reduction in surface heating. However, for the current test model configuration and flow conditions, the change in wall temperature has a significant impact on both the surface 
heat flux and flow structure where the heating rate actually increases along the expansion surface (Fig. 2(a)), but is reduced along most of the compression surface. Furthermore, the extent of separation, indicated by the region of negative shear stress, is noticeably increased (Fig. 2(b)) in that there is a region of separated flow where separation

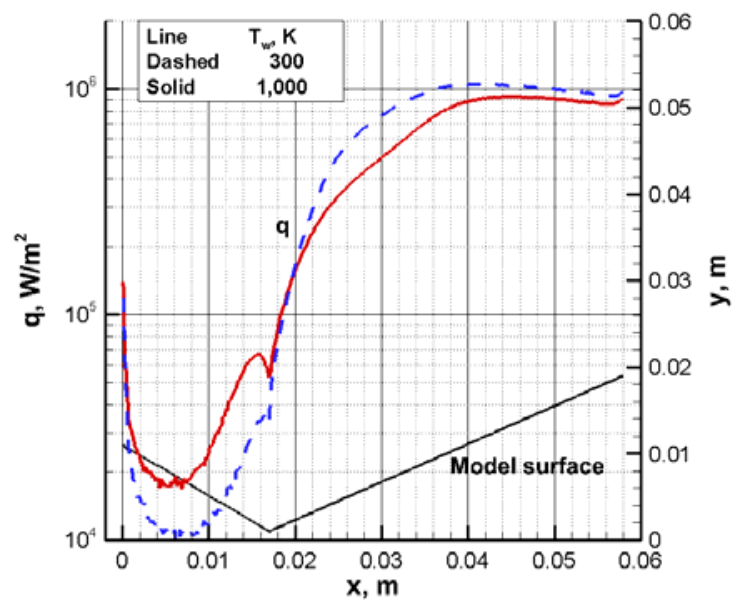

(a) Heat flux

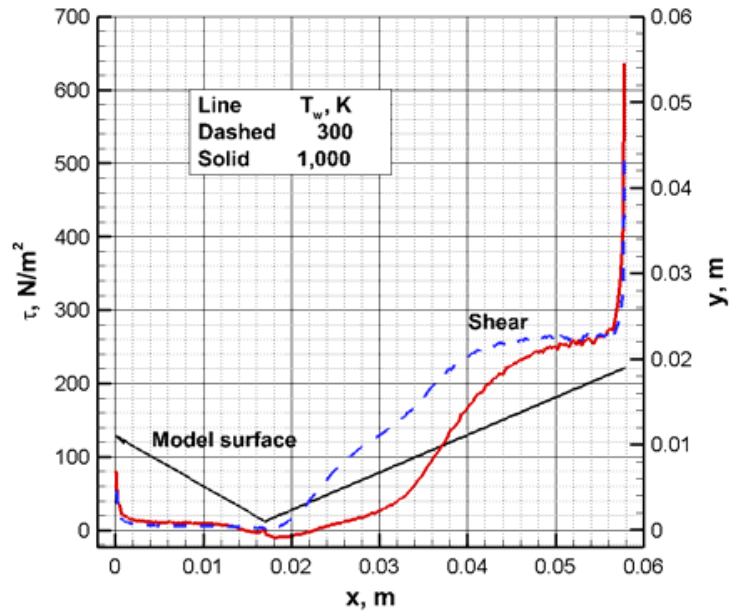

(b) Shear stress

FIGURE 2. Effect of wall temperature on (a) heat flux and (b) shear stress along the model surface

occurs on the expansion surface $\left(\mathrm{X}_{\mathrm{S}}=14.65 \mathrm{~mm}\right)$ and reattachment occurs on the compressions surface $\left(\mathrm{X}_{\mathrm{r}}=22.60\right.$ $\mathrm{mm}$ ). For the increased heating along the expansion surface at the higher wall temperature, we find that the flow in this region is more energetic with an increase in both surface heat flux and pressure (which is not presented). For example, $5 \mathrm{~mm}$ from the leading edge where the surface pressure achieves a minimum value, the surface pressure for the $T_{\mathrm{w}}=1,000 \mathrm{~K}$ condition is 2.2 times the corresponding value for $\mathrm{T}_{\mathrm{w}}=300 \mathrm{~K}$. Consequently, the current results indicate that potential experiments at hot wall conditions would have a measurable impact on both the flowfield structure and surface flux.

The impact of the surface boundary conditions for vibrational energy accommodation was investigated since some experimental evidence suggests [12] that when there is significant vibrational nonequilibrium within the flow domain, as is the case for the 'tick' configuration, the vibrational energy does not accommodate to the surface temperature when the molecules impact the surface. The potential impact of surface vibrational accommodation was investigated by examining the simulated results for both full and zero accommodation of the vibrational energy for surface temperatures of $300 \mathrm{~K}$ and $1,000 \mathrm{~K}$. These results (not shown) indicate that the heating rate is reduced slightly (maximum effect of about 6 percent on the compression surface and less on the expansion surface) when compared to corresponding results for full accommodation. The impact of the vibrational energy accommodation boundary condition on surface pressure and on the locations and extent of separation is negligible; a finding that is in agreement with the results presented in Ref. [11] for hypersonic separated flows about biconic and hollow cylinder-flare configurations.

\section{Results for Different ‘Tick’ Model Configurations}

All results presented and discussed up to this point have been for the initial 'tick' configuration as depicted in Fig. 1(b) except for the effect of rounding the leading edge with radii of 0.10 and $0.20 \mathrm{~mm}$. As discussed in Ref. [1] (see Fig. 5 of Ref [1]), the departure from the sharp leading edge has a significant impact on the flow in that the extent of separation increases from essentially zero for the sharp leading edge to values of 11.6, 28.6, and $39.8 \mathrm{~mm}$ for leading edge radii of $0.05,0.10$, and $0.20 \mathrm{~mm}$, respectively (corresponding locations for separation are: 13.6, 8.5, and $5.1 \mathrm{~mm}$, respectively, when measured from the beginning of the flat expansion surface, where the length of the expansion surface remained constant with leading edge rounding). Consequently, the extent of separation becomes larger as the leading edge radius is increased and has a significant impact on the surface flux and degree of rarefaction experienced by the flow. As an example, the surface pressure along the expansion surface at $\mathrm{x}=5.0 \mathrm{~mm}$ 
(approximate location for the minimum pressure for the sharp leading edge model) increases substantially with increasing nose radii. The magnitude of the pressure increase with respect to that for the sharp leading edge was 10 , 18 , and 102 for nose radii of $0.05,0.10$, and $0.20 \mathrm{~mm}$, respectively. Obviously, there is a substantial change in the heating and skin friction distributions as previously shown in Ref. [1] for the two larger nose radii conditions. Also, note that these simulations did not have the truncated forebody as shown in Fig. 1(b). Instead, the forebody extended to a location such that position 4, as listed in Fig. 1(b), was located at $y=0.00$ and " $x$ " at the appropriate position to achieve the stated forebody slope.

More recent DSMC simulations have been made to complement the progress being made in the design and fabrication of test hardware; including results for models taken from initial CAD drawings as well as the actual fabricated hardware. Figures 3 and 4 present data for these various configurations along with results from parametric studies that were made to explore some trends that were not obvious at first inspection. Since the scope of computational test cases was expanding, the decision was made to perform this series of preliminary simulations for a four species nonreacting gas model consisting of only the freestream species, and the number of simulation molecules was approximately half the values used previously; that is, approximately 4.5 million simulated molecules with about 0.5 million collision cells. The assumption that an average $\mathrm{mcs} / \mathrm{mfp}$ value of about 0.27 is adequate for the present study appears to be justified when the surface flux quantities are compared with the corresponding results for a mcs/mfp value of 0.18 , and there are negligible differences in the two sets of results.

First, some comments concerning the fabricated test model and how it differs from the initial configuration (Fig. 1(b)). The model design is one in which the length of the expansion surface, inclined $-30^{\circ}$ to the horizontal, can be adjusted to any value between 14.80 and $19.88 \mathrm{~mm}$ for a fixed expansion surface whose length is $50 \mathrm{~mm}$ and inclined $24.15^{\circ}$ to the horizontal. The leading edge bevel angle is $20.12^{\circ}$; therefore, the model's forward-facing surface is inclined $-50.12^{\circ}$ to the flow. Increasing the bevel angle by almost a factor of two over that of the initial configuration previously studied has a noticeable impact on the flow environment, as will be detailed shortly. As for the fabricated model leading edge, the radius has been determined to be $0.01 \mathrm{~mm}$. Figure 3(b) presents a sketch of the fabricated model dimensions and surface boundary conditions for which DSMC simulations were made, assuming that the leading edge is sharp (length of expansion surface increased by $0.01 \mathrm{~mm}$ ). Results for a leading edge radius of $0.01 \mathrm{~mm}$ have not been obtained at this time due to numerical round off issues; however, results are included for a radius of $0.10 \mathrm{~mm}$.

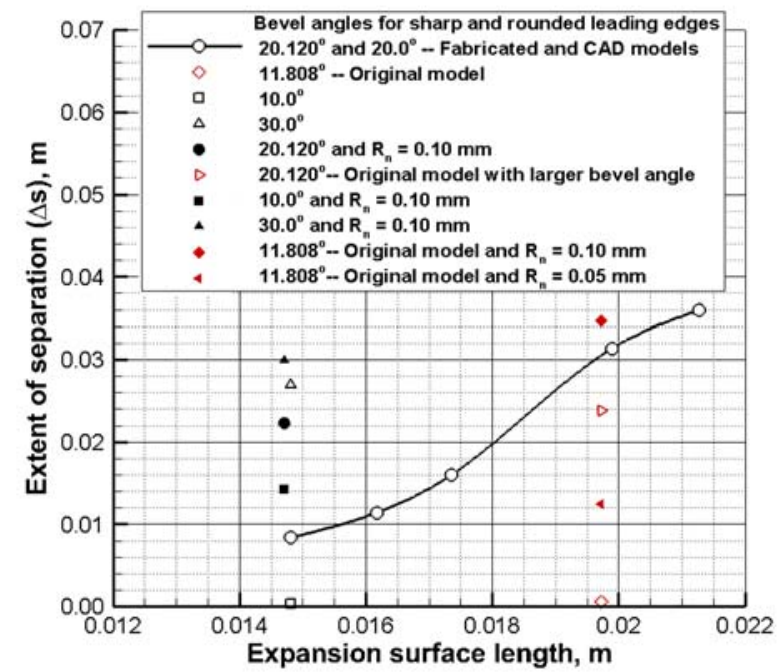

(a) Impact of test model configuration on separation

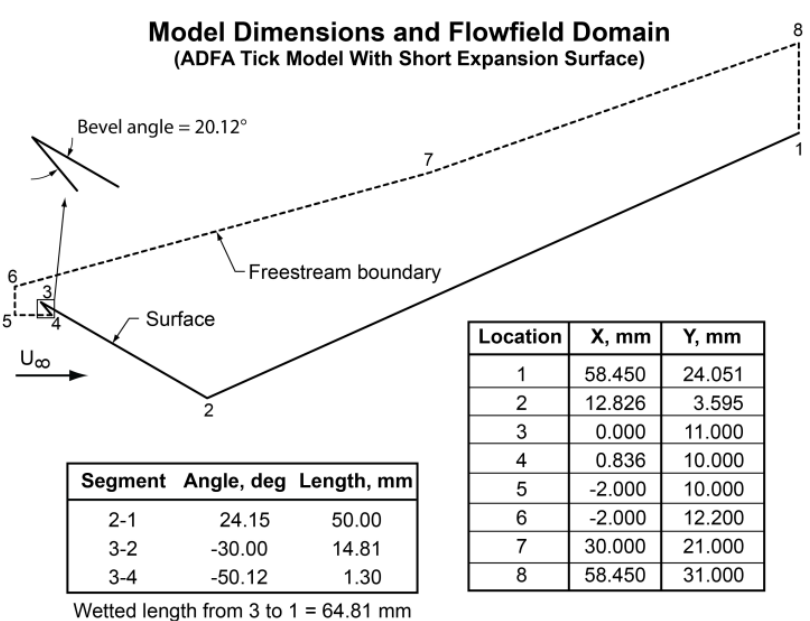

(b) Sharp'tick' model with short expansion surface

FIGURE 3. Results for DSMC, 4-species, nonreacting simulations and geometric description for one configuration

Figure 3(a) provides a summary of the results for the effects of several model parameters, where the separation distance (in terms of the wetted distance along the model surface, $\Delta s$ ) is plotted as a function of the model expansion surface length. Focusing first on the five open circle data points connected by a solid line that represents the data for both the CAD and fabricated models, the data shows that the extent of separation increases as the model size 
increases, a trend that is expected (larger Reynolds number, larger extent of separation). These results are for sharp leading edge models and the three data points along the solid curve for the fabricated model are as follows: minimum expansion length surface (first open circle from the left), maximum length expansion surface (the fourth open circle from the left), and the midpoint expansion length surface (the third open circlet from the left).

Since the results for the fabricated model with the longest expansion surface indicated a substantial separated flow region, the question arose as to why there was a large difference between these results and that for the original test model (data point for original test model is in the lower right corner of Fig. 3(a)); that is, essentially no separation evident for a model with similar physical dimensions. A comparison of surface results for heating rate and shear stress for these two simulations is presented in Fig. 4(a), highlighting the large differences in heating along the expansion surface and the major difference in extent of separation.

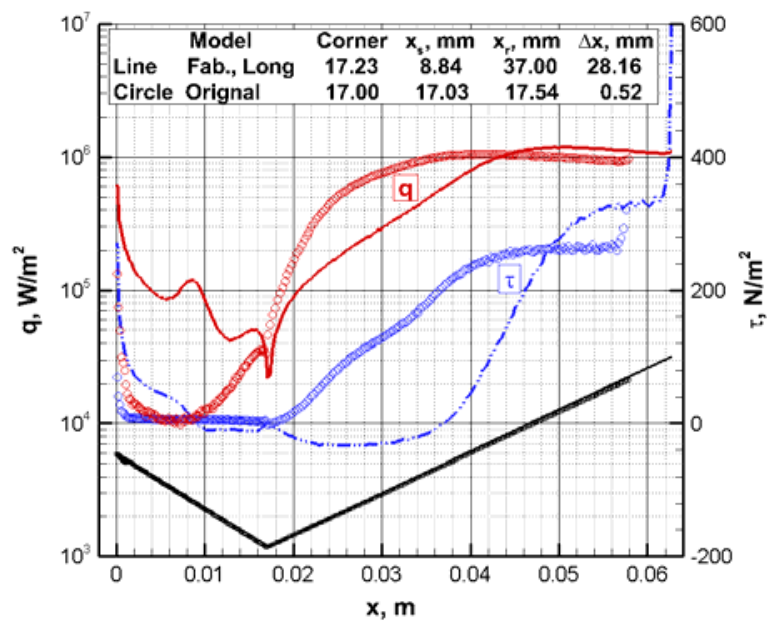

(a) Original and current long model results, both sharp

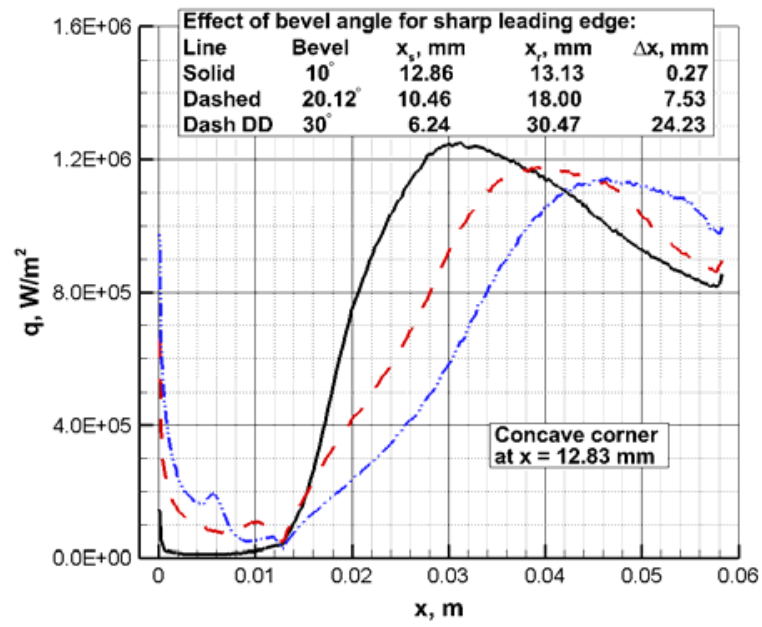

(b) Impact of model bevel angle-short expansion

FIGURE 4. Impact of surface geometry on surface flux

When the flowfield data was examined for the two cases, one could see that the larger bevel angle was producing an obvious impact on both the leading edge and downstream flowfield. By conducting a parametric study where simulations are made for sharp leading edges with bevel angles of 10, 20.12, and 30 degrees (with the forwardfacing surface of the model inclined at 40, 50.12 and 60 degrees to the freestream direction, respectively), we produce data that provides an indication of the effect. As the leading edge bevel angle is increased, a stronger bow shock and a stronger shock/shock interaction occur above the compression surface and higher pressure is generated on the expansion surface, which produces less rarefied conditions. The DSMC simulations were performed for the test model with the short expansion surface, and the surface heating distributions for these cases are shown in Fig. 4(b) while the separation extent is depicted in Fig. 3(a) as open symbols. Also shown in Fig. 3(a) is the result for the initial configuration modified so that its leading edge bevel angle was the same as that of the current test model (increased from 11.8 degrees to 20.12 degrees). For this increase in the bevel angle, the extent of separation increased from $0.57 \mathrm{~mm}$ to $24 \mathrm{~mm}$.

Also included in Fig. 3(a) are several data points showing the effect of incorporating small leading edge radii on both the original 'tick' model and that for the current short expansion surface model. As evidenced by the results for the short expansion surface, the impact of a $0.10 \mathrm{~mm}$ leading edge radius becomes less for the larger bevel angle cases. Such a trend would be expected as increasing the leading edge radius or the leading edge bevel angle appears to have similar effects on the flow.

Surface distributions for heating and pressure along with tabulated data for the magnitude of separated flow are presented in Fig. 4(c) for the fabricated test model for three different expansion surface lengths: the shortest, the longest, and a length equal the average of the short and long expansion surfaces. The heating and pressure distributions for the three configurations are very similar for the first $5 \mathrm{~mm}$ where both heating and pressure decreases rapidly as the flow expands. Following the initial expansion, the distributions begin to assume different features because of the three different separated flow regions ( $\Delta \mathrm{x}$ values of 7.5, 14.4, and $28.2 \mathrm{~mm}$ ) corresponding to the three different expansion surface lengths. 
Each of the heating distributions (Fig. 4(c)) has either one or two local maxima followed by a decrease to a minimum value at the model's corner (intersection of expansion and compression surfaces), and then continues to increase rapidly along the compression surface until a maximum value occurs downstream of flow reattachment but well before the end of the model. The maximum heating location moves downstream as the length of the expansion surface and the size of the separated region increases. As for the first local heating rate maximum along the expansion surface, the shape is attributed to the fact that the surface pressure begins to increase before separation with a corresponding increase in heating rate. Then the first local maximum occurs since the heating decrease as separation occurs.

The reason for the second heating maximums along the expansion surface (evident in all of the subfigures of Fig. 4) is not known; however, there is no evidence of multiple vortices.

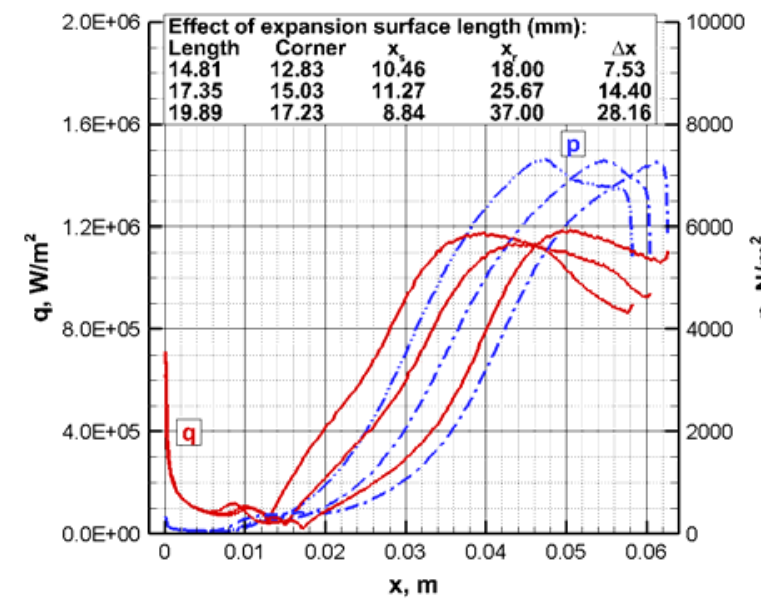

(c) Effect of expansion surface length on heating

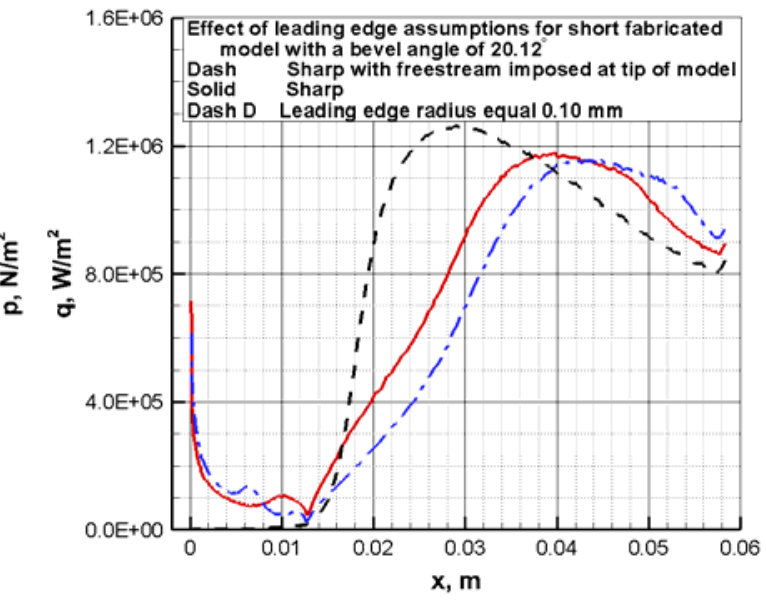

(d) Effect of leading edge conditions on heating

FIGURE 4. . Impact of surface geometry on surface flux -- concluded

The heating and pressure distributions observed for the 'tick' configuration (Fig. 4(c)) are very similar to the general trends observed for pressure and heating distributions from experimental studies reported in the past [3, 13]. Also, the current results clearly support Chapman's [3] original observation that the leading edge separation is a limiting case of the often studied compression corner flow but with minimal boundary layer effects.

The general trends observed for heating and pressure distributions from experimental studies [13] based on laminar hypersonic flows with separation coincides closely with the trends observed for the current 'tick' simulations (Fig. 4(c)). First, the separation position occurs near the location of the first inflection point (maximum slope) of the initial pressure rise and is also the location at which the heat transfer rate decreases significantly. Second, the pressure reaches a plateau for a sufficiently large separation region, while the heat transfer is significantly reduced. Third, at or preceding the intersection of the two surfaces, the heat transfer experiences a minimum and then increases rapidly, as does the pressure, with increasing distance along the second surface. The current results for the 'tick' configuration show that peak heating and peak pressure occurs well downstream of reattachment, indicating that the recompression is a gradual process for these reattaching laminar shear layers.

As previously discussed for the 'tick' configuration, the general features of the leading edge are such that the extent to which the leading edge is rounded and the magnitude of its bevel angle have a significant impact on the type of flow realized. Futhermore, the way in which the numerical simulation is set up can alter the results substantially as demonstrated in Fig. 4(d). Results for three surface heating distibutions are presented for the short expansion surface model: one for a sharp leading edge, one for a leading edge with a radius of $0.10 \mathrm{~mm}$, and one for a sharp leading edge where the inflow boundary condition is at the tip of the sharp leading edge. For the latter case, the calculated heating along the expansion surface is quite small, but increases with distance along the expansion surface and then rises sharply along the compression surface while reaching a maximum value that is both greater in magnitude and located further upstream compared with the simulated results where the inflow boundary condition is positioned upstream of the leading edge. 
The observed differences in results for the two sharp leading edge simulations due to where the inflow boundary is positioned drive home the point of how sensitive these flows can be to the characterization and modeling of the leading edge; issues important to recognize for meaningful experimental and computational comparisons. Results of the current simulations indicate that any 'tick' model configuration will be influenced by the leading edge for the flow conditions investigated. The extent of this effect becomes more pronounced as either the leading edge departs from the idealized sharp state or the bevel angle is increased. (Note how the heating distribution for the sharp leading edge results with a 10 degree bevel angle (Fig. 4(b)) is approaching the general features of that for the simulation with the freestream conditons imposed at the tip of the model (Fig. 4(d)).)

\section{CONCLUSIONS}

Results of a computational study are presented for hypersonic air flow about 'tick' configurations where flow separation, rarefaction, and thermal nonequilibrium are important features of the flow. Experiments are being organized for the 'tick' configuration in the T-ADFA free-piston shock tunnel located at The University of NSW, Canberra, Australia. Numerical simulations are made with the DSMC code of Bird for one of the more rarefied flow conditions available in T-ADFA, and a range of test model configurations are investigated with particular attention focused on the role of the model's leading edge (extent of rounding and the size of the leading edge bevel angle), length of expansion surface relative to fixed compression surface, effect of wall temperature, and surface recombination assumptions for the vibrational energy. Key findings of this study are evidence of strong sensitivity of the flow to features of the leading edge design and also that the imposition of a hot wall boundary condition produces appreciable changes in both the extent of separation and the surface flux values relative to that for a cold wall.

\section{ACKNOWLEDGMENTS}

Dr. O'Byrne and Prof. Gai would like to acknowledge the US Air Force Asian Office of Aerospace Research and Development and the Australian Research Council (Grant DP140100842) for their support of this research. The authors would like to thank Prof. G. A. Bird for his assistance with details of his DS2V code, and for allowing us to use this code.

\section{REFERENCES}

1. J. N. Moss, S. O’Byrne, N. R. Deepak, and S. L. Gai, "Simulations of Hypersonic, High-Enthalpy Separated Flow over a 'Tick' Configuration”, Rarefied Gas Dynamics, 28 ${ }^{\text {th }}$ International Symposium, Mareschal and Santos eds., American Institute of Physics, Conference proceedings 1501,Vol. 2, Melville, New York, 2012, pp. 1453-1460.

2. N. R. Deepak, S. L. Gai, J. N. Moss, and S. O’Byrne, “Hypersonic High-Enthalpy Flow in a Leading-edge Separation” Proceedings of the $29^{\text {th }}$ International Shock Wave Symposium, July, 2013, University of Wisconsin, Madison, USA.

3. D. R. Chapman, D. M. Kuehn, and H. K. Larson, "Investigation of Separated Flows in Subsonic and Supersonic Streams with Emphasis on the Effect of Transition”, NACA Technical Report 1356, 1958.

4. R. Hruschka, S. O’Byrne, and H. H. Kleine, "Comparison of Velocity and Temperature Measurements with Simulations in a Hypersonic Wake Flow”, Exp. Fluids, 51(2), 407-421 (2011). DOI: 10.1007/s00348-11-1039-9.

5. R. Hruschka, "Optical Studies and Simulations of Hypervelocity Flow Fields around Blunt Bodies: PhD Thesis, University of NSW, 2010.

6. M. K. McIntosh, "Computer Program for the Numerical Calculation of Frozen and Equilibrium Conditions in Shock Tunnels”, Australian National University Technical Report, 1968.

7. I. M. Vardavas, "Modeling Reactive Gas Flows within Shock Tunnels”, Australian Journal of Physics, 37, pp. 157-177, 1983.

8. K. N. C. Bray, "Chemical and Vibrational Nonequilibrium in Nozzle Flows: in Nonequilibrium Flows, Part II, Marcel Dekker, New York, 1970.

9. G. A. Bird, Molecular Gas Dynamics and the Direct Simulation of Gas Flows, Oxford: Clarendon Press, 1994.

10. G. A. Bird, http://gab.com.au/index.html

11. J. N. Moss and G. A. Bird, "DSMC Simulations of Hypersonic Flows with Shock Interactions and Validation with Experiments”. AIAA Journal, 43(12), pp. 2565-2573 (2005).

12. J. R. Meloans and I. Graur, “New Thermal Conditions at the Wall in High Speed Flows,” Rarefied Gas Dynamics, 23rd International Symposium, Ketsdever and Muntz, eds., American Institute of Physics, 2003, CD-ROM.

13. D. Needham and J. Stollery, “Boundary Layer Separation in Hypersonic Flow,” AIAA Paper 66-455, Jan. 1966. 